\title{
ON THE GROWTH OF DERIVATIVES OF FUNCTIONS OMITTING TWO VALUES
}

BY

\author{
A. S. GAlbRAith, W. SEIDEL, AND J. L. WALSH
}

1. In an earlier paper by two of the authors $\left({ }^{1}\right)$ relations were obtained between $D_{p}(w)$ on the one hand and expressions of the form $\left(1-|z|^{2}\right)^{p}\left|f^{(p)}(z)\right|$ on the other, where $w=f(z)$ is a more or less arbitrary function analytic in $|z|<1$ and $D_{p}(w)$ is the radius of $p$-valence defined below in $\S 2$. In particular, results were obtained for functions $f(z)$ omitting two values. The most important of these results were the following two:

First, if $f(z)$ is analytic in the unit circle $|z|<1$ and omits two finite values there, and if $\left\{z_{n}\right\} \quad\left(\left|z_{n}\right|<1\right)$ is a sequence of points such that $w_{n}=f\left(z_{n}\right)$ is bounded, then

$$
\lim _{n \rightarrow \infty} D_{p}\left(w_{n}\right)=0
$$

is necessary and sufficient for

$$
\lim _{n \rightarrow \infty}\left(1-\left|z_{n}\right|^{2}\right)^{k}\left|f^{(k)}\left(z_{n}\right)\right|=0, \quad k=1,2, \cdots, p .
$$

It can be shown by examples $\left({ }^{2}\right)$ that if the boundedness of $w_{n}$ is not assumed, the theorem ceases to be true. To treat this case it was shown that:

Second, if $f(z)$ is analytic in the unit circle $|z|<1$ and omits two finite values there, if $\left\{z_{n}\right\}\left(\left|z_{n}\right|<1\right)$ is a sequence of points such that $w_{n}=f\left(z_{n}\right)$ becomes infinite with $n$, and if there exists a number $\epsilon>0$ such that

$$
\lim _{n \rightarrow \infty}\left|w_{n}\right|^{(1+\epsilon)\left(2^{p}-1\right)} D_{p}\left(w_{n}\right)=0
$$

then

$$
\lim _{n \rightarrow \infty}\left(1-\left|z_{n}\right|^{2}\right)^{k}\left|f^{(k)}\left(z_{n}\right)\right|=0, \quad k=1,2, \cdots, p .
$$

It is the object of the present paper to furnish an extension of the second of these results. With the aid of a result of L. H. Loomis $\left({ }^{3}\right)$ in the case $p>1$

Presented to the Society, October 30, 1948; received by the editors September 3, 1947, and, in revised form, September 28, 1948.

(1) W. Seidel and J. L. Walsh, On the derivatives of functions analytic in the unit circle and their radii of univalence and p-valence, Trans. Amer. Math. Soc. vol. 52 (1942) pp. 128-216; in particular Chap. IV. This paper will be referred to as SW.

(2) SW, Chap. IV, §23.

(3) L. H. Loomis, On an inequality of Seidel and Walsh, Bull. Amer. Math. Soc. vol. 48 (1942) pp. 908-911. 
we are able to replace condition (1) by the lighter condition $\left(^{4}\right)(p>0)$

$$
\lim _{n \rightarrow \infty}\left|w_{n}\right|^{p}\left(\log \left|w_{n}\right|\right)^{p} D_{p}\left(w_{n}\right)=0 .
$$

In a recent paper $\left(^{5}\right)$ Rogosinski has studied sufficient conditions for the approach to zero of expressions similar to $\left(1-\left|z_{n}\right|^{2}\right)^{p}\left|f^{(p)}\left(z_{n}\right)\right|$. His conditions, while leading to simpler proofs, do not cover all the cases considered here and in SW.

2. We turn now to the definition of the radius of $p$-valence $D_{p}(w)$. Let $C_{p}$ be a simply-connected Riemann configuration containing the point $w_{0}$, lying over the circle $\left|w-w_{0}\right|<\rho$ and covering it precisely $p$ times. Then $C_{p}$ is called a $p$-sheeted circle with center $w_{0}$ and radius $\rho$. Now let $w=f(z)$, regular in the unit circle $|z|<1$, map the unit circle on a Riemann configuration $R$. Let $w_{0}$ be an arbitrary point belonging to $R$. A non-negative number $D_{p}\left(w_{0}\right)$, called the radius of p-valence of $R$ at the point $w_{0}$, shall be associated with the point $w_{0}$ in the following manner:

(a) For $p=1$, we define $D_{p}\left(w_{0}\right)=D_{1}\left(w_{0}\right)$ as the radius of univalence of $R$ at the point $w_{0}$, which is zero if $w_{0}$ is a branch point of $R$ and otherwise is equal to the radius of the largest smooth circle with center at $w_{0}$ and lying in $R$;

(b) If there exists a $p$-sheeted circle with center $w_{0}$ contained in $R$, there exists a largest such circle, and the radius of this largest circle is defined as $D_{p}\left(w_{0}\right)$;

(c) If $p$ is greater than 1 , and if $w_{0}$ is a branch point of order greater than $p-1$, then $D_{p}\left(w_{0}\right)=0$;

(d) If there exists no $p$-sheeted circle $(p>1)$ with center $w_{0}$ contained in $R$, and if $w_{0}$ is not a branch point of order greater than $p-1$, then we define $\left(^{6}\right)$ $D_{p}\left(w_{0}\right)$ as $D_{p-1}\left(w_{0}\right)$.

3. LEMMA 1. Let $f(z)$ be regular in $|z|<1$ and let $|f(z)|$ be not greater than $M$ in $|z|<\beta(<1)$. Then setting $w_{0}=f(0)$, we have

$$
\left|f^{\prime}(0)\right| \leqq\left(8 M D_{1}\left(w_{0}\right) / \beta^{2}\right)^{1 / 2} .
$$

This lemma is an immediate consequence of Theorem 3, Chapter II, of SW, if $f(z)$ is replaced by $f(\beta z)$ there. Here $D_{1}\left(w_{0}\right)$ may refer to the image of $|z|<1$ under either of the transformations $w=f(z)$ and $w=f(\beta z)$.

THEOREM 1. Let $f(z)$ be regular in $|z|<1$ and omit two finite values there. Let $\left\{z_{n}\right\}$ be an infinite sequence of points interior to $|z|<1$ such that, setting

(4) The authors are indebted to the referee for improving Theorem 1 below and for materially simplifying the proofs of both Theorems 1 and 2 .

(5) W. W. Rogosinski, On the order of the derivatives of a function analytic in an angle, J. London Math. Soc. vol. 20 (1945) pp. 100-109.

(6) For a detailed discussion of the properties of $D_{p}(w)$ see SW, Chap. II. 
$w_{n}=f\left(z_{n}\right)$, we have

$$
\lim _{n \rightarrow \infty} w_{n}=\infty
$$

and

$$
\lim _{n \rightarrow \infty}\left|w_{n}\right|\left(\log \left|w_{n}\right|\right) D_{1}\left(w_{n}\right)=0
$$

Then

$$
\lim _{n \rightarrow \infty}\left(1-\left|z_{n}\right|^{2}\right)\left|f^{\prime}\left(z_{n}\right)\right|=0 .
$$

It entails no loss of generality to let the omitted values be 0 and $e$, since a linear transformation will carry any two finite omitted values $a$ and $b$ into 0 and $e$, and (3) persists. Let

$$
\phi_{n}(\zeta)=e^{i \alpha_{n}} f\left(\left(\zeta+z_{n}\right) /\left(1+\bar{z}_{n} \zeta\right)\right), \quad-\pi<\alpha_{n} \leqq \pi,
$$

where $\alpha_{n}$ is so chosen that $\phi_{n}(0)=\left|w_{n}\right|$. The functions $f\left(\left(\zeta+z_{n}\right) /\left(1+\bar{z}_{n} \zeta\right)\right)$ in $|\zeta|<1$ omit the values 0 and $e$ for every $n$, and consequently form a normal family $\left({ }^{7}\right)$. The functions $\phi_{n}(\zeta)$ also form a normal family, for from the indices of a given sequence a subsequence can be chosen such that the corresponding $\alpha_{n}$ approach a limit; the corresponding $f\left(\left(\zeta+z_{n}\right) /\left(1+\bar{z}_{n} \zeta\right)\right)$ will contain a subsequence converging uniformly in any closed subregion of the unit circle.

Hence $\phi_{n}(\zeta) \rightarrow \infty$ with $n$, uniformly in $|\zeta| \leqq 1 / 2$. Then we may assume $n$ so large that $\left|\phi_{n}(\zeta)\right|>1$ in $|\zeta| \leqq 1 / 2$. Now let

$$
g_{n}(\zeta)=\log \phi_{n}(\zeta), \quad|\zeta| \leqq 1 / 2,
$$

choosing that branch of the logarithm which makes $g_{n}(0)=\log \left|w_{n}\right|$ real and positive. The real part of $g_{n}(\zeta)$ is positive. Then by Carathéodory's inequality $\left({ }^{8}\right)$

$$
\left|g_{n}(\zeta)\right| \leqq 3 g_{n}(0), \quad|\zeta| \leqq 1 / 4
$$

Using this in Lemma 1 , we have $\left(^{9}\right)$

$$
\left|g_{n}^{\prime}(0)\right| \leqq C\left\{g_{n}(0) D_{1}\left(g_{n}(0)\right)\right\}^{1 / 2},
$$

where $C$ is a constant independent of $n$. Here $D_{1}\left(g_{n}(0)\right)$ refers to the Riemann configuration which is the image of $|\zeta|<1 / 4$.

By the mapping (6) the circle $g_{n}(0)+t D_{1}\left(g_{n}(0)\right),|t|<1$, in the Riemann configuration into which the function $g_{n}(\zeta)$ maps $|\zeta|<1 / 2$, is mapped in to the set

(7) P. Montel, Leçons sur les familles normales de fonctions analytiques, Paris, 1927, p. 61.

(8) See, for example, C. Carathéodory, Conformal representation, Cambridge, 1932, p. 44, inequality (74.3).

(9) We may assume that $g_{n}(0)$ is not a branch point, since $g_{n}^{\prime}(0)=0$ implies $f^{\prime}\left(z_{n}\right)=0$. 


$$
\exp \left\{g_{n}(0)+t D_{1}\left(g_{n}(0)\right)\right\}=\phi_{n}(0)\left\{1+t D_{1}\left(g_{n}(0)\right)+\cdots\right\}
$$

in the Riemann configuration into which the function $\phi_{n}(\zeta)$ maps $|\zeta|<1 / 2$. Now, $D_{1}\left(g_{n}(0)\right)$ must approach zero as $n$ becomes infinite, by (3) and the properties of the logarithmic map (6).

Since $D_{1}\left(g_{n}(0)\right) \rightarrow 0$, for sufficiently large $n$ the set (8) contains the circle about $\phi_{n}(0)$ with radius $2^{-1} \phi_{n}(0) D_{1}\left(g_{n}(0)\right)$, so that

$$
D_{1}\left(w_{n}\right) \geqq 2^{-1} \phi_{n}(0) D_{1}\left(g_{n}(0)\right) \text {. }
$$

Hence, from (7),

$$
\left|g_{n}^{\prime}(0)\right| \leqq C^{\prime}\left\{g_{n}(0) D_{1}\left(w_{n}\right) / \phi_{n}(0)\right\}^{1 / 2},
$$

$C^{\prime}$ a constant independent of $n$. If in this last formula we substitute $g_{n}(0)$ $=\log \left|w_{n}\right|, \phi_{n}(0)=\left|w_{n}\right|, g_{n}^{\prime}(0)=\phi_{n}^{\prime}(0) / \phi_{n}(0)=\left(1-\left|z_{n}\right|^{2}\right) f^{\prime}\left(z_{n}\right) /\left|w_{n}\right|$, we get

$$
\left(1-\left|z_{n}\right|^{2}\right)\left|f^{\prime}\left(z_{n}\right)\right| \leqq C^{\prime}\left|w_{n}\right|\left\{\log \left|w_{n}\right| D_{1}\left(w_{n}\right) /\left|w_{n}\right|\right\}^{1 / 2} \text {, }
$$

and a reference to hypothesis (3) establishes (4).

Theorem 1 can be generalized to derivatives of any order. In fact, we prove the following theorem:

THEOREM 2. Let $f(z)$ be regular in $|z|<1$ and omit two finite values there. Let $\left\{z_{n}\right\}$ be an infinite sequence of points interior to $|z|<1$ such that, setting $w_{n}=f\left(z_{n}\right)$,

$$
\lim _{n \rightarrow \infty} w_{n}=\infty,
$$

and such that there is a positive integer $p$ for which

$$
\lim _{n \rightarrow \infty}\left|w_{n}\right|^{p}\left(\log \left|w_{n}\right|\right)^{p} D_{p}\left(w_{n}\right)=0 .
$$

Then

$$
\lim _{n \rightarrow \infty}\left(1-\left|z_{n}\right|^{2}\right)^{k}\left|f^{(k)}\left(z_{n}\right)\right|=0, \quad k=1,2, \cdots, p .
$$

The theorem will be proved by induction. It is true for $p=1$ by Theorem 1 , and we shall assume that it holds for $p-1$.

As in Theorem 1 the functions $\phi_{n}(\zeta)$ and $g_{n}(\zeta)$ are defined by (5) and (6), respectively, and the proof proceeds as before until we reach inequality (7), which is replaced by $\left({ }^{10}\right)$

(12) $\left|g_{n}^{\prime}(0)\right|+\frac{\left|g_{n}^{\prime \prime}(0)\right|}{2 !}+\cdots+\frac{\left|g_{n}^{(p)}(0)\right|}{p !} \leqq K_{p}\left\{D_{p}\left(g_{n}(0)\right)\right\}^{1 /(p+1)}\left(g_{n}(0)\right)^{p /(p+1)}$, where $D_{p}\left(g_{n}(0)\right)$ is the radius of $p$-valence at $g_{n}(0)$ of the Riemann configura-

(10) Loomis, op. cit., inequality (2). 
tion $R_{\theta_{n}}$ on which $g_{n}(\zeta)$ maps the circle $|\zeta| \leqq 1 / 4$, and $K_{p}$ is a constant independent of $n$.

We may assume that $g_{n}(0)$ is not a branch point of order greater than $p-1$, since $g_{n}^{(\mathbf{k})}(0)=0$ for $k=1,2, \cdots, p$ would imply that $f^{(k)}\left(z_{n}\right)=0$ for $k=1,2, \cdots, p$. Hence, $D_{p}\left(g_{n}(0)\right)$ may be taken positive. There exists then, for some integer $k, 1 \leqq k \leqq p$, a $k$-sheeted circle $C_{k}\left(g_{n}(0)\right)$ about $g_{n}(0)$ with radius $D_{p}\left(g_{n}(0)\right)$. The points of each sheet of $C_{k}\left(g_{n}(0)\right)$ will lie over the circle represented by the expression $g_{n}(0)+t D_{p}\left(g_{n}(0)\right),|t|<1$. The transformation (6) carries $C_{k}\left(g_{n}(0)\right)$ into a $k$-sheeted, simply connected subregion $S_{k}\left(\phi_{n}(0)\right)$ of the Riemann configuration $R_{\phi_{n}}$ on which $\phi_{n}(\zeta)$ maps the circle $|\zeta| \leqq 1 / 4$. Each sheet of $S_{k}\left(\phi_{n}(0)\right)$ will lie over the region defined by the expression

$$
\exp \left\{g_{n}(0)+t D_{p}\left(g_{n}(0)\right)\right\}=\phi_{n}(0)\left\{1+t D_{p}\left(g_{n}(0)\right)+\cdots\right\}, \quad|t|<1 .
$$

As in the proof of Theorem 1 , it is readily seen that $\lim _{n \rightarrow \infty} D_{p}\left(g_{n}(0)\right)=0$. Consequently, for sufficiently large $n$ the region $S_{k}\left(\phi_{n}(0)\right)$ will contain, for some integer $k^{\prime} \leqq k$, a $k^{\prime}$-sheeted circle $\Gamma_{k^{\prime}}\left(\phi_{n}(0)\right)$ about $\phi_{n}(0)$ with radius $2^{-1} \phi_{n}(0) D_{p}\left(g_{n}(0)\right)$, so that

$$
\begin{aligned}
2^{-1} \phi_{n}(0) D_{p}\left(g_{n}(0)\right) & \leqq D_{k^{\prime}}\left(w_{n}\right) \\
& \leqq D_{p}\left(w_{n}\right)
\end{aligned}
$$

since $D_{p}\left(w_{n}\right)$ is monotonically nondecreasing with $p$. This, with inequality (12), yields the inequality

$$
\begin{aligned}
\left|g_{n}^{\prime}(0)\right|+\frac{\left|g_{n}^{\prime \prime}(0)\right|}{2 !}+\cdots+ & \frac{\left|g_{n}^{(p)}(0)\right|}{p !} \\
& \leqq C\left\{D_{p}\left(w_{n}\right) / \phi_{n}(0)\right\}^{1 /(p+1)}\left(\log \phi_{n}(0)\right)^{p /(p+1)}
\end{aligned}
$$

for a suitable constant $C$, independent of $n$.

Differentiation of the function $g_{n}(\zeta)$ given in (6) shows that for each $k \leqq p, g_{n}^{(k)}(0)$ consists of $\phi_{n}^{(k)}(0) / \phi_{n}(0)$ plus a finite sum of fractions which, aside from constant factors, contain in the numerators only products of $\phi_{n}^{\prime}(0)$, $\phi_{n}^{\prime \prime}(0), \cdots, \phi_{n}^{(k-1)}(0)$, and in the denominators only positive powers of $\phi_{n}(0)$ not greater than the $k$ th. Now $\left({ }^{11}\right)$

$$
\frac{\phi_{n}^{(k)}(0)}{k !}=\sum_{j=1}^{k}(-1)^{k-i} C_{k-1, k-j} \bar{z}_{n}^{k-j} \frac{\left(1-\left|z_{n}\right|^{2}\right)^{i} f^{(j)}\left(z_{n}\right)}{j !} .
$$

But because $D_{k-1}(w) \leqq D_{k}(w)$, hypothesis (10) of Theorem 2 and the inductive hypothesis imply that

$$
\lim _{n \rightarrow \infty}\left(1-\left|z_{n}\right|^{2}\right)^{j} f^{(j)}\left(z_{n}\right)=0, \quad j=1,2, \cdots, p-1 .
$$

(i1) SW, Chap. I, §2, Lemma 2. 
Hence, by (14),

$$
\lim _{n \rightarrow \infty} \phi_{n}^{(k)}(0)=0, \quad k=1,2, \cdots, p-1 .
$$

If we now multiply inequality (13) by $\phi_{n}(0)=\left|w_{n}\right|$, it will follow from (14), (15), and (16) that all the terms in the left member of the inequality so obtained will approach zero as $n$ tends to infinity, except perhaps the term $\left(1-\left|z_{n}\right|^{2}\right)^{p}\left|f^{(p)}\left(z_{n}\right)\right| / p$ !. But as $n$ becomes infinite, the right member of this inequality will approach

$$
C \cdot \lim _{n \rightarrow \infty}\left\{\left|w_{n}\right|^{p}\left(\log \left|w_{n}\right|\right)^{p} D_{p}\left(w_{n}\right)\right\}^{1 /(p+1)} .
$$

Therefore, by (10)

$$
\lim _{n \rightarrow \infty}\left(1-\left|z_{n}\right|^{2}\right)^{p}\left|f^{(p)}\left(z_{n}\right)\right|=0
$$

which proves Theorem 2 .

4. The theorems of Rogosinski, in the paper referred to earlier, have simpler proofs than the similar theorems given here and in SW, but the conditions imposed upon the functions are unnecessarily restrictive. On the other hand, the condition that the function omit two values, made in our paper as well as in SW and in the paper by Rogosinski, is actually essential to the conclusion, as the following example, which is similar to one given in SW, Chap. IV, §23, Remark 2, shows.

Let

$$
f(z)=\exp \{i /(1-z)\}-i /(1-z)
$$

so that

$$
f^{\prime}(z)=[\exp \{i /(1-z)\}-1] \cdot i /(1-z)^{2} .
$$

We have $f^{\prime}(z)=0$ when $z=\beta_{n}=1-1 / 2 n \pi$, so that the branch points of the Riemann configuration are

$$
B_{n}=f\left(\beta_{n}\right)=1-2 n \pi i .
$$

Let

$$
z_{n}=1-1 /\left(2 n \pi+\alpha_{n}\right),
$$

where $\left\{\alpha_{n}\right\}$ is a sequence of positive numbers to be specified later, with $\lim _{n \rightarrow \infty} \alpha_{n}=0$. Then

$$
w_{n}=f\left(z_{n}\right)=e^{i \alpha_{n}}-2 n \pi i-i \alpha_{n} .
$$

To compute $D_{1}\left(w_{n}\right)$ it is not sufficient to compute $\left|w_{n}-B_{n}\right|$, because $w_{n}$ and $B_{n}$ may conceivably be on different sheets of the Riemann configuration. 
However, the segment of the real axis joining $z_{n}$ and $\beta_{n}$ is mapped by $w=f(z)$ on an analytic arc, of length $L$, say, joining $w_{n}$ and $B_{n}$, and $D_{1}\left(w_{n}\right) \leqq L$. But

$$
L=\int_{\beta_{n}}^{z_{n}}\left|f^{\prime}(x)\right| d x .
$$

Now $\left|f^{\prime}(x)\right|$ is easily computed to be $\pm \sin (1 / 2(1-x)) \cdot 2 /(1-x)^{2}$, so that, for $n$ sufficiently large,

$$
L= \pm 8 \sin \left(n \pi+\alpha_{n} / 4\right) \sin \left(\alpha_{n} / 4\right)
$$

and

$$
D_{1}\left(w_{n}\right) \leqq 2^{-1} \alpha_{n}^{2} .
$$

Since $\alpha_{n}$ approaches zero, $\left|w_{n}\right|\left(\log \left|w_{n}\right|\right)$ is $O(n \log n)$ for large $n$. On the other hand

$$
\left(1-\left|z_{n}\right|^{2}\right)\left|f^{\prime}\left(z_{n}\right)\right|=\left|e^{i \alpha_{n}}-1\right|\left(1+\left|z_{n}\right|\right)\left(2 n \pi+\alpha_{n}\right),
$$

which is, except for a factor bounded away from zero, $n \alpha_{n}$. Thus if, for example, $\alpha_{n}=n^{-3 / 4}$, hypothesis (3) of Theorem 1 is satisfied, but the conclusion of the theorem is false.

This example shows that one of the two important hypotheses of our theorems cannot be dropped, and also shows that if the function $f(z)$ does not omit two values, (3) cannot be replaced by the stronger condition

$$
\lim _{n \rightarrow \infty}\left|w_{n}\right|{ }^{1+\epsilon} D_{1}\left(w_{n}\right)=0, \quad 0<\epsilon<1 / 2 .
$$

Whether the other hypothesis, on the rate at which $D_{p}\left(w_{n}\right)$ approaches zero, is required the authors do not know. The example of Seidel and Walsh referred to above shows that (3) cannot be replaced by

$$
\lim _{n \rightarrow \infty}\left|w_{n}\right|^{\gamma} D_{1}\left(w_{n}\right)=0, \quad 0<\gamma<1,
$$

but the necessity of the factor $\log \left|w_{n}\right|$ is an open question.

Aberdeen Proving Ground,

AberdeEn, MD.

UNIVERSITY OF ROCHESTER,

ROCHESTER, N. Y.

INSTITUTE FOR NUMERICAL ANALYSIS,

Los ANgeles, Calif.

HARVARD UNIVERSITY,

Cambridge, Mass. 